\title{
Perfil lipídico como factor de riesgo cardiovascular y su relación con la actividad física en adultos que frecuentan el Parque de la Salud del Instituto de Previsión Social
}

LIPID PROFILE AS A FACTOR OF CARDIOVASCULAR RISK FACTOR AND ITS RELATIONSHIP WITH PHYSICAL ACTIVITY IN ADULTS WHO EXERCISE AT the Health Park from Instituto de Prevision Social - Public Welfare Hospital

\author{
Aguilar Rabito, Ana ${ }^{1}$ \\ Romero Ausfeld, María Josée \\ Torres López, Andrea Monserrat ${ }^{3}$
}

${ }^{1}$ Docente y Coordinadora de Postgrados en Nutrición de la Facultad de Ciencias Químicas de la Universidad Nacional de Asunción.

${ }^{2}$ Nutricionista colaboradora en el Departamento de Alimentación y Nutrición en el Hospital de Clínicas de la Universidad Nacional de Asunción.

${ }^{3}$ Nutricionista en Consultorio Soporte y Asesoramiento de SA del Hospital Privado San Lucas S.A. 


\section{RESUMEN}

Introducción: Las enfermedades cardiovasculares representan la principal causa de morbi-mortalidad en el mundo occidental. La mayor parte de la evidencia muestra que el ejercicio aeróbico tiene un efecto favorable sobre el perfil lipídico y el riesgo de enfermedades cardiovasculares. Objetivo: Evaluar la relación entre el gasto calórico de la actividad física y el perfil lipídico como factor de riesgo cardiovascular en adultos de ambos sexos que frecuentan el Parque de la Salud del Instituto de Previsión Social. Materiales y métodos: Observacional analítico de corte transverso realizado en 98 sujetos con mediciones de peso, talla, índice de masa corporal, circunferencia de cintura, presión arterial, perfil lipídico, glucemia y gasto calórico de la actividad física, considerándola leve, moderada e intensa, $<600,600-1.499 \mathrm{y} \geq 1500$ METs respectivamente. Resultados: $48,3 \%$ de los hombres y $32,7 \%$ de las mujeres presentaron malnutrición por exceso. Según la medición de circunferencia de cintura, el $31,6 \%$ de los hombres y mujeres presentaron riesgo cardiovascular. Se encontró relación positiva entre la actividad física y el colesterol-HDL $(\mathrm{p}=0,038)$ y negativa con los triglicéridos $(\mathrm{p}=0,040)$ y colesterol-VLDL $(\mathrm{p}=0,041)$. Al discriminar por sexo, en las mujeres se encontró relación positiva solo con el colesterol-HDL $(\mathrm{p}=0,027)$ mientras que en los hombres se halló una relación positiva con el colesterol-HDL $(\mathrm{p}=0,002)$ y una relación negativa con el colesterol total $(\mathrm{p}=0,018)$, colesterol- $\mathrm{LDL}$ $(\mathrm{p}=0,039)$, triglicéridos y colesterol-VLDL $(\mathrm{p}=0,014)$. Conclusiones: Si bien el tamaño muestral fue mínimo, estos resultados reflejan lo mencionado en diversas bibliografías, si la actividad física es realizada de manera constante, además de tener efectos benéficos sobre el colesterol total, colesterol LDL y colesterol HDL, también produce un incremento de la actividad lipolítica y consecuentemente una disminución de los niveles plasmáticos de triglicéridos, de esta manera se pueden prevenir factores de riesgos cardiovasculares asociados al sedentarismo como son las dislipidemias. Se dispone de suficiente evidencia científica acerca de los efectos del ejercicio aeróbico sobre el perfil lipídico destacando el cambio favorable que ejerce en el metabolismo lipoproteico.

Palabras claves: enfermedades cardiovasculares; gasto calórico de la actividad física; perfil lipídico.

\section{UNiBE- ${ }_{\text {Esudios envestigaciones }}^{\text {REVISTA }}$}




\section{ABSTRACT}

Introduction: Cardiovascular diseases are the main cause of morbiditymortality in the western world. Levels of high LDL-cholesterol, low HDL-cholesterol, hypertension, smoking and physical inactivity are risk factors involved in the occurrence of cardiovascular events. Objective: Evaluate the relation between the caloric spent of a physical activity and the lipid profile as cardiovascular risk factor in adults of both sexes, that haunt the "Parque de la Salud del Instituto de Prevision Social". Materials and methods: Observational analytic of transverse cut conducted in 98 subjects with anthropometric and blood pressure measurements were carried out, together with lipid profile and glucose determination. Results: $48.3 \%$ of the male subjects presented malnutrition by excess, the same for female in a $32.7 \%$. According the waistline perimeter it was stand a similar cardiovascular risk for female and male subjects in $31.6 \%$. Significant positive relation was found between physical activity and the HDL-Cholesterol ( $\mathrm{p}=0.038$ ), triglycerides $(\mathrm{p}=0.04)$, and VLDLCholesterol $(\mathrm{p}=0.041)$ was found. According to sex, affected HDLcholesterol $(\mathrm{p}=0,027)$ in women, and total cholesterol $(\mathrm{p}=0,018)$, HDLcholesterol $(\mathrm{p}=0,002)$, LDL-cholesterol $(\mathrm{p}=0,039)$, triglycerides and VLDL-cholesterol $(\mathrm{p}=0,014)$ in men. Conclusion: When the physical activity by category of intensity was analyzed, both men and women performed low and moderate physical activity, showing an altered lipid profile. The most of the subjects spent more than $700 \mathrm{Kcal} /$ week, which supposes a cardiovascular benefit. Inadequate values of lipid profile were found more frequently in men than women.

Keywords: cardiovascular diseases, caloric spent of activities, lipid profile. 


\section{INTRODUCCIÓN}

Las enfermedades cardiovasculares (ECV) son un conjunto de enfermedades que afectan el corazón y vasos sanguíneos que lo rodean. Se caracterizan por el daño de la pared arterial coronaria y posterior depósito de grasa, también conocido como ateroma, produciendo estrechamiento, engrosamiento y ruptura de las arterias. ${ }^{4}$

En los países desarrollados las enfermedades cardiovasculares son la primera causa de muerte y van en aumento, considerándose un importante problema de salud pública para estos países. En Paraguay la proporción de muertes por enfermedades cardiovasculares en el año 2005, fue del $22 \%$ y se previó, con un análisis estadístico, que para el 2010 aumentaría al $28 \%$ y en el 2020 , al $30 \% .^{5.6}$

La mayor parte de las enfermedades cardiovasculares tienen una base genética. Sin embargo, son los estilos de vida los que determinan que la patología se desarrolle a lo largo de los años. La alimentación inadecuada, el tabaquismo, el alcoholismo, el sedentarismo y el estrés son factores de riesgo que contribuyen al desarrollo de la ateroesclerosis y el consiguiente aumento de las enfermedades cardiovasculares. ${ }^{7,}$, De todas las muertes que se producen cada año en el mundo, un $30 \%$ son atribuibles a las ECV. Se estima que en 2010 fallecieron por estas causas 18,1 millones de personas, de las cuales el $80 \%$ vivía en países de ingresos bajos y medianos. ${ }^{9}$

Se considera actividad física (AF) a cualquier movimiento corporal ocasionado por la contracción muscular produciendo como resultado un gasto energético. Esta se asocia al menor riesgo de ECV, enfermedad

\footnotetext{
${ }^{4}$ Hernández MA, García HL. Factores de riesgo y protectores de enfermedades cardiovasculares en población estudiantil universitaria. Rev. Fac. Med. 2007; 30 (2): 119-123.

${ }^{5}$ Escobar MC, Zacarías I. Programa de actividad física para la prevención y control de los factores de riesgo cardiovascular. Instituto de Nutrición y Tecnología de los Alimentos (INTA) de la Universidad de Chile, Comité de Prevención de Riesgo Cardiovascular de la Sociedad de Cardiología y Cirugía Cardiovascular y la Sociedad de Kinesiología en Cardiología y Cirugía Cardiovascular, Organización Panamericana de la Salud/Organización Mundial de la Salud. Santiago. 2004.

${ }^{6}$ Ministerio de Salud Pública y Bienestar Social. Enfermedades cardiovasculares constituirán $30 \%$ de causas de muerte en el 2020, según estimaciones del INPCARD. Disponible en: http://www.mspbs.gov.py

${ }^{7}$ Escobar MC, Zacarías I., 2004.

${ }^{8}$ Onzari M. Fundamentos de Nutrición en el Deporte. $1^{\mathrm{a}}$ ed. Buenos Aires: El Ateneo; 2008. p. 328 .

${ }^{9}$ OMS. Enfermedades cardiovasculares. Ginebra: Organización Mundial de la Salud; 2011.
}

120 uniBe $\left.\right|_{\text {Estudios elnvestigaciones }} ^{\text {REVISTA }}$


coronaria, accidente vascular encefálico, mortalidad cardiovascular y total. Se ha estimado que el sedentarismo podría ser responsable de $12,2 \%$ de los infartos al miocardio en la población mundial y la realización de actividad física una de las principales formas de prevenirla. ${ }^{10,11}$

Los beneficios de la AF se han atribuido principalmente a la disminución del índice de masa corporal (IMC), de la resistencia a la insulina, al aumento del colesterol HDL y mejoría de la hipertensión y función endotelial, junto con una disminución de los niveles de factores inflamatorios, como la proteína C-reactiva ultrasensible (PCRus) ${ }^{12,13}$

Si bien no existe claridad absoluta acerca de los mecanismos por los cuales la AF ejerce protección cardiovascular, se demuestra que los sujetos con mayor nivel de AF y potencia aeróbica máxima presentan menores factores de riesgo cardiovascular clásicos y emergentes. ${ }^{14}$

Según todo lo anterior, el propósito del presente estudio es identificar cómo influye el gasto calórico de la actividad física en la variabilidad del perfil lipídico como factor de riesgo cardiovascular, considerando que el infarto de miocardio es la causa más común de muerte por aterosclerosis, enfermedad que ataca a las arterias en forma generalizada pudiéndose presentar distintos síndromes clínicos (infarto de miocardio, accidente cerebrovascular, aneurisma de aorta, angina de pecho, gangrena, insuficiencia cardiaca y muerte súbita). ${ }^{15,16}$

${ }^{10}$ Onzari M, 2008.

${ }^{11}$ Fundación del corazón. Com. Fundación Española de Cardiología. Factores de riesgo cardiovascular. Málaga. Fundación Española del Corazón; [actualizada 27 ene 2011; citado 20 ago 2011]. Disponible en: http://www.fundaciondelcorazon.com/prevencion/riesgocardiovascular.html

${ }^{12}$ Imizcoz MA. La hipertensión arterial como factor de riesgo de enfermedad cardiovascular. Anales de la Facultad de Navarra; 21 (1).

${ }^{13}$ Leal E, Aparicio D, Luti Y, Acosta L, Finol F, Rojas E, et al. Actividad física y enfermedad cardiovascular. Revista Latinoamericana de Hipertensión. 2009; 4 (1): 3-17.

${ }^{14}$ Onzari M., 2008.

${ }^{15}$ Ibíd.

${ }^{16}$ Disponible en: http://www.who.int/mediacentre/factsheets/fs317/es/index.html 


\section{MATERIALES Y MÉTODOS}

Estudio transversal analítico con muestreo no probabilístico por conveniencia, en el que participaron 98 sujetos que frecuentaron el Parque de la Salud del Instituto de Previsión Social durante los meses de setiembre y octubre del 2011. Se excluyó a aquellas personas medicadas con antihipertensivos, hipoglucemiantes e hipolipemiantes y mujeres embarazadas. La participación al estudio fue de forma voluntaria, siendo protegida la privacidad y la confidencialidad de los datos.

Los datos fueron recolectados mediante una encuesta adaptada para el estudio basándose en el modelo de la Versión Panamericana de la Salud del método "STEPwise" de la Organización Mundial de la Salud para la vigilancia de los factores de riesgo de las enfermedades crónicas, aplicado mediante entrevista. ${ }^{17} \mathrm{El}$ instrumento consta de información demográfica, mediciones del comportamiento que incluye: tabaquismo, alcohol, dieta, actividad física, mediciones físicas: estatura y peso, circunferencia de cintura, presión arterial y mediciones bioquímicas: glucemia, lípidos en sangre como triglicéridos, colesterol total, colesterol-HDL, colesterolLDL. Las muestras de sangre fueron recolectadas luego de 8-12 horas de ayuno y antes de realizar actividad física.

Primero se aplicó la encuesta y luego se procedió a medir las variables objetivas relacionadas a la valoración antropométrica y la presión arterial conforme estándares internacionales.

Para la medición antropométrica se utilizó una báscula con tallímetro incorporado de marca Balmak con una capacidad máxima de 150 kilogramos y 2 metros, con precisión de 100 gramos y $10 \mathrm{~mm}$; y una cinta métrica antropométrica marca IsoSource cuya unidad mínima es 1 cm y máxima 1,5 metros. Para la medición de la presión arterial se utilizó el esfigmomanómetro manual.

La actividad física se evaluó a través de la "sección principal: actividad física" del cuestionario, donde se debía registrar: a) Las veces por semana y el tiempo que se utiliza para desplazarse caminando; b) La realización de deportes intensos en el tiempo libre especificando el deporte, (Ej.: correr, jugar al futbol, ir al gimnasio) las veces por semana y durante cuánto tiempo lo realiza; c) La realización de actividad de intensidad

${ }^{17}$ Barreda R, Santos J, Sakuray S, Villanueva J, Delgado I, Avendaño M. Prevalencia de factores de riesgo de enfermedades cardiovasculares en mayores de 18 años de los distritos de Tacna, Gregorio Alabarracin, Ciudad Nueva, Tarata y Candarave 2007; (78): 1-43.

\section{UNIBE $\mid \begin{aligned} & \text { REVISTA } \\ & \text { CIENTÍFICA }\end{aligned}$


moderada (Ej.: caminar de prisa, ir en bicicleta, nadar) las veces por semana y durante cuánto tiempo lo realiza. El índice de sedentarismo se clasificó en horas que pasa sentado o recostado en un día, considerando sedentario $>2 \mathrm{~h} /$ día y no sedentario $\leq 2 \mathrm{~h} /$ día. $^{18}$

La intensidad del ejercicio se obtuvo mediante el gasto calórico de las actividades o METs, que corresponde al gasto energético durante la actividad, medido como múltiplos de la tasa de metabolismo basal. Para ello se determinó el gasto energético por AF según múltiplos del MB, de acuerdo a la edad, sexo, peso corporal. La intensidad de la actividad se clasificó según criterios del IPAQ en leve $(<600)$, moderada (600-1.499) e intensa $(y \geq 1500)$ METs. ${ }^{19,20}$

Los datos fueron digitalizados en una planilla electrónica de $®$ Microsoft Office Excel Versión 2007 (EE.UU.) y ®SPSS para Windows. Versión 11.5 (EE.UU.).

Se aplicó la Prueba de Kolmogorov-Smirnov para verificar la distribución de los datos. Los datos cualitativos son expresados en frecuencia absoluta (n) y relativa (\%), y los cuantitativos en promedio y desviación estándar para variables homogéneas y medianas con los valores límites para aquellas de distribución no homogénea, todos estratificados por sexo. Para evaluar diferencias según tipo de variables, se utilizó la prueba $\mathrm{Chi}^{2}$ $\left(X^{2}\right)$, o prueba t según corresponda al tipo de variables; considerándose un nivel de significancia estadística de $p \leq 0,05$. La prueba de hipótesis fue realizada mediante la Correlación de Pearson para variables cuantitativas.

Aspectos éticos de confidencialidad, respeto, beneficencia y justicia fueron considerados durante la investigación, y el estudio contó con la aprobación del Comité de Ética de la Facultad de Ciencias Químicas de la Universidad Nacional de Asunción.

${ }^{18}$ Boraita A. La práctica deportiva mejora el perfil lipídico plasmático, pero ¿a cualquier intensidad? Rev. Esp. Cardiol. 2004; 57 (6): 495-498.

${ }^{19}$ Hernández MA, García HL, 2007.

${ }^{20}$ Ulate G,FernándezA. Relaciones del perfil lipídico con variables dietéticas, antropométricas, bioquímicas y otros factores de riesgo cardiovascular en estudiantes universitarios. AMC. 2001; 43(2): 70-6. 


\section{RESULTADOS}

Se incluyeron en el estudio 98 sujetos con edades comprendidas entre 19 y 64 años ( $43 \pm 13$ años \pm 13 años), de los cuales 48 (49\%) eran hombres (edad promedio: 44 años) y $50(51 \%)$ eran mujeres (edad promedio: 42 años).

En cuanto al nivel de educación, la mayoría refirió tener estudios universitarios $52,1 \%(\mathrm{n}=52)$, siendo levemente mayor el porcentaje de hombres con estudios universitarios respecto a las mujeres. Se clasificó el nivel de ingresos en menos del salario mínimo, entre uno y dos salarios mínimos y más de dos salarios mínimos, pudiendo observar que la mayor parte de los hombres refirió tener un ingreso de más de dos salarios mínimos 29,6\% (n=29) a diferencia de las mujeres quienes refirieron tener un ingreso de entre uno y dos salarios mínimos $17,3 \%(n=17)$.

En lo que respecta al hábito de fumar y tipo de fumador, se encontró que la mayoría de los encuestados no tienen el hábito de fumar $90,8 \%$ $(n=89)$, del 9,2\% ( $n=9)$ restante, $30 \%(n=30)$ de los hombres y $60 \%$ $(n=6)$ de las mujeres eran fumadores leves con un consumo promedio de 10 cigarrillos por día, y solo $10 \%(\mathrm{n}=1)$ de los hombres consumía más de 10 cigarrillos por día siendo el consumo moderado.

Referente al consumo de bebidas alcohólicas, se constató que el 69,4\% $(n=68)$, de los encuestados las consumía, destacándose un mayor consumo en hombres que en mujeres. El promedio de edad de inicio de consumo de alcohol fue de $14 \pm 11$ años.

Al evaluar la frecuencia de consumo de frutas y verduras se observó similar consumo tanto en hombres como en mujeres siendo la frecuencia de consumo de dos frutas por día, cuatro veces a la semana y tres porciones de verduras por día, cinco veces a la semana.

$\mathrm{Al}$ medir los factores de riesgo cardiovascular, se observaron valores significativamente superiores en hombres en lo que respecta a circunferencia de cintura, triglicéridos, colesterol-VLDL, presión arterial sistólica y diastólica, no así el colesterol-HDL que fue significativamente mayor en mujeres. No se encontraron diferencias estadísticas en ambos sexos en cuanto al índice de masa corporal, colesterol total, glicemia e índice de sedentarismo (Tabla 1).

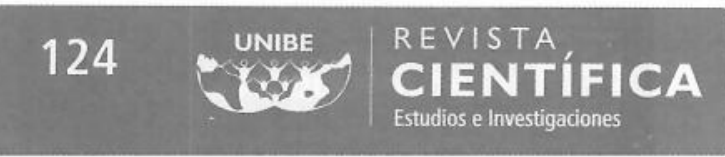


Tabla 1. Factores de riesgo cardiovascular por sexo

\begin{tabular}{|c|c|c|c|c|c|}
\hline & Sexo & Mínimo & Máximo & Promedio $\pm \mathrm{DE}$ & $\mathbf{p}$ \\
\hline \multirow{2}{*}{$\operatorname{IMC}\left(\mathbf{k g} / \mathbf{m}^{2}\right)$} & Varones & 22 & 38 & $29,1 \pm 3,8$ & \multirow{2}{*}{0,225} \\
\hline & Mujeres & 17 & 44 & $27,8 \pm 6,4$ & \\
\hline \multirow{2}{*}{$\mathrm{CC}(\mathrm{cm})$} & Varones & 80 & 127 & $101,0 \pm 10,4$ & \multirow{2}{*}{$<0,0001$} \\
\hline & Mujeres & 62 & 125 & $85,5 \pm 14,9$ & \\
\hline \multirow{2}{*}{ PAS (mmHg) } & Varones & 80 & 200 & $121,7 \pm 20,9$ & \multirow{2}{*}{0,006} \\
\hline & Mujeres & 80 & 180 & $110,6 \pm 18,2$ & \\
\hline \multirow{2}{*}{ PAD (mmHg) } & Varones & 50 & 140 & $81,3 \pm 15,4$ & \multirow{2}{*}{0,005} \\
\hline & Mujeres & 50 & 100 & $73,4 \pm 10,1$ & \\
\hline \multirow{2}{*}{$\begin{array}{l}\text { Glucemia (mg/ } \\
\text { dl) }\end{array}$} & Varones & 66 & 172 & $83,5 \pm 19,5$ & \multirow{2}{*}{0,639} \\
\hline & Mujeres & 60 & 274 & $81,2 \pm 29,7$ & \\
\hline \multirow{2}{*}{ C-Total (mg/dl) } & Varones & 134 & 290 & $204,9 \pm 34,6$ & \multirow{2}{*}{0,133} \\
\hline & Mujeres & 100 & 277 & $193,4 \pm 40,5$ & \\
\hline \multirow{2}{*}{ C-HDL (mg/dl) } & Varones & 30 & 74 & $46,7 \pm 10,7$ & \multirow{2}{*}{$<0,0001$} \\
\hline & Mujeres & 29 & 82 & $55,8 \pm 12$ & \\
\hline \multirow{2}{*}{ C-LDL (mg/dl) } & Varones & 55 & 201 & $121,5 \pm 33,1$ & \multirow{2}{*}{0,094} \\
\hline & Mujeres & 35 & 173 & $110,2 \pm 33$ & \\
\hline \multirow{2}{*}{ TG (mg/dl) } & Varones & 71 & 449 & $183,4 \pm 93,2$ & \multirow{2}{*}{0,013} \\
\hline & Mujeres & 38 & 368 & $137,3 \pm 86,1$ & \\
\hline \multirow{2}{*}{$\begin{array}{l}\text { C-VLDL (mg/ } \\
\text { dl) }\end{array}$} & Varones & 14 & 90 & $36,8 \pm 18,7$ & \multirow{2}{*}{0,012} \\
\hline & Mujeres & 3 & 74 & $27,4 \pm 17,5$ & \\
\hline \multirow{2}{*}{$\begin{array}{l}\text { Índice de } \\
\text { Sedentarismo }\end{array}$} & Varones & 1 & 12 & $5,8 \pm 2,8$ & \multirow[b]{2}{*}{0,383} \\
\hline & Mujeres & 1 & 12 & $5,3 \pm 2,5$ & \\
\hline
\end{tabular}

Prueba t de Student

Al analizar el comportamiento del perfil lipídico ante la intensidad mediante MET, en general se encontró relación significativa y positiva entre la actividad física y el colesterol-HDL, y una relación negativa y significativa con los triglicéridos y colesterol-VLDL. Al discriminar por sexo, en las mujeres se encontró relación significativa y positiva solo con el colesterol-HDL. En los hombres se halló una relación positiva y significativa con el colesterol-HDL y una relación negativa y significativa con el colesterol total, colesterol- LDL, triglicéridos y colesterol-VLDL (Tabla 2). 
Tabla 2. Comportamiento del perfil lipídico ante la intensidad según MET

\begin{tabular}{|c|c|c|c|c|c|c|}
\hline \multicolumn{2}{|c|}{ General } & $\begin{array}{l}\text { C-Total } \\
\text { (mg/dl) }\end{array}$ & $\begin{array}{l}\text { C-HDL } \\
(\mathrm{mg} / \mathrm{dl})\end{array}$ & $\begin{array}{l}\text { C-LDL } \\
(\mathrm{mg} / \mathrm{dl})\end{array}$ & $\begin{array}{c}\mathrm{TG} \\
(\mathrm{mg} / \mathrm{dl})\end{array}$ & $\begin{array}{c}\text { C-VLDL } \\
(\mathrm{mg} / \mathrm{dl})\end{array}$ \\
\hline \multirow{2}{*}{$\begin{array}{l}\text { Intensidad de } \\
\text { la AF (MET. } \\
\text { Min/semana) }\end{array}$} & Pearson & $-0,174$ & $0,210^{*}$ & $-0,159$ & $-0,208^{*}$ & $-0,207^{*}$ \\
\hline & $\begin{array}{l}\text { Sig. } \\
\text { (bilateral) }\end{array}$ & 0,087 & 0,038 & 0,117 & 0,040 & 0,041 \\
\hline \multicolumn{7}{|l|}{ Mujeres } \\
\hline \multirow{2}{*}{$\begin{array}{l}\text { Intensidad de } \\
\text { la AF (MET. } \\
\text { Min/semana) }\end{array}$} & Pearson & $-0,104$ & $0,313^{*}$ & $-0,109$ & $-0,246$ & $-0,251$ \\
\hline & $\begin{array}{l}\text { Sig. } \\
\text { (bilateral) }\end{array}$ & 0,473 & 0,027 & 0,453 & 0,085 & 0,079 \\
\hline \multicolumn{7}{|l|}{ Varones } \\
\hline \multirow{2}{*}{$\begin{array}{l}\text { Intensidad de } \\
\text { la AF (MET. } \\
\text { Min/semana) }\end{array}$} & Pearson & $-0,341 *$ & $0,435^{* *}$ & $-0,299 *$ & $-0,351^{*}$ & $-0,351$ \\
\hline & $\begin{array}{l}\text { Sig. } \\
\text { (bilateral) }\end{array}$ & 0,018 & 0,002 & 0,039 & 0,014 & 0,014 \\
\hline
\end{tabular}

* La correlación de Pearson es significante al nivel 0,05 (bilateral)

** La correlación de Pearson es significante al nivel 0,01 (bilateral)

En cuanto a la asociación de la intensidad de la actividad física con el perfil lipídico según sexo, se observó que la mayoría de los hombres al igual que las mujeres $(\mathrm{H}=40 ; \mathrm{M}=46)$ realizaron actividad física de intensidad leve presentando los hombres en su mayoría niveles inadecuados de colesterol total, triglicéridos y colesterol-LDL, a diferencia de las mujeres que a la misma intensidad presentaron solo niveles de colesterol-LDL inadecuados.

Aintensidad moderada ( $\mathrm{H}=7 ; \mathrm{M}=4)$, la mayoría de los hombres presentaron un perfil de colesterol-LDL inadecuado, no así las mujeres que a la misma intensidad presentaron en su mayoría el perfil lipídico adecuado.

Se observó que 1 hombre realizó actividad física intensa presentando un adecuado perfil lipídico.

Al analizar el perfil lipídico en hombres, se comprobó que cuanto mayor era la concentración de colesterol total, los niveles de colesterolLDL eran significativamente más altos. Aquellos con valores elevados de colesterol-HDL presentaron valores significativamente más bajos de TG y colesterol-VLDL. Los hombres con TG elevados presentaron valores significativamente más bajos de colesterol-HDL y niveles significativamente más altos de colesterol-VLDL (Tabla 3).

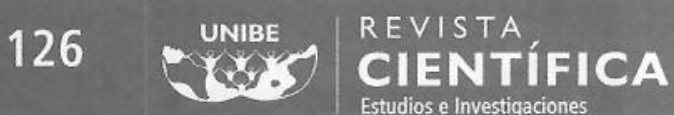


Tabla 3. Comportamiento del perfil lipídico en varones

\begin{tabular}{|c|c|c|c|c|c|c|}
\hline \multicolumn{2}{|c|}{ VARONES } & $\begin{array}{l}\text { C-Total } \\
(\mathrm{mg} / \mathrm{dl})\end{array}$ & $\begin{array}{l}\text { C-HDL } \\
(\mathrm{mg} / \mathrm{dl})\end{array}$ & $\begin{array}{l}\text { C-LDL } \\
(\mathrm{mg} / \mathrm{dl})\end{array}$ & $\begin{array}{c}\text { TG } \\
(\mathrm{mg} / \mathrm{dl})\end{array}$ & $\begin{array}{c}\text { C-VLDL } \\
(\mathrm{mg} / \mathrm{dl})\end{array}$ \\
\hline \multirow{2}{*}{$\begin{array}{l}\text { Col.T } \\
(\mathrm{mg} / \mathrm{dl})\end{array}$} & Pearson & 1 & 0,083 & $0,893^{\text {*** }}$ & 0,225 & 0,222 \\
\hline & Sig. (bilateral) & & 0,576 & 0,0001 & 0,125 & 0,129 \\
\hline \multirow{2}{*}{$\begin{array}{l}\text { C-HDL } \\
(\mathrm{mg} / \mathrm{dl})\end{array}$} & Pearson & 0,083 & 1 & 0,068 & $-0,538 * *$ & $-0,539 * *$ \\
\hline & Sig. (bilateral) & 0,576 & & 0,647 & 0,0001 & 0,0001 \\
\hline \multirow{2}{*}{$\begin{array}{l}\text { C-LDL } \\
(\mathrm{mg} / \mathrm{dl})\end{array}$} & Pearson & $0,893^{* *}$ & 0,068 & 1 & $-0,155$ & $-0,158$ \\
\hline & Sig. (bilateral) & 0,0001 & 0,647 & & 0,292 & 0,284 \\
\hline \multirow{2}{*}{$\begin{array}{l}\mathrm{TG} \\
(\mathrm{mg} / \mathrm{dl})\end{array}$} & Pearson & 0,225 & $-0,538^{* * *}$ & $-0,155$ & 1 & $1,000 * *$ \\
\hline & Sig. (bilateral) & 0,125 & 0,0001 & 0,292 & & 0,0001 \\
\hline \multirow{3}{*}{$\begin{array}{l}\text { C-VLDL } \\
(\mathrm{mg} / \mathrm{dl})\end{array}$} & Pearson & 0,222 & $-0,539 * *$ & $-0,158$ & $1,000 * *$ & 1 \\
\hline & Sig. (bilateral) & 0,129 & 0,0001 & 0,284 & 0,0001 & \\
\hline & $\mathrm{N}$ & 48 & 48 & 48 & 48 & 48 \\
\hline
\end{tabular}

* La correlación de Pearson es significante al nivel 0,05 (bilateral)

** La correlación de Pearson es significante al nivel 0,01 (bilateral)

Al analizar el perfil lipídico en mujeres, se comprobó que cuanto mayor era la concentración de colesterol total, los niveles de colesterol-LDL, TG y colesterol-VLDL eran significativamente más altos. Aquellas con valores elevados de colesterol-HDL presentaron valores significativamente más bajos de TG y colesterol-VLDL. Las mujeres con TG elevados presentaron valores significativamente más altos de colesterol total y de colesterol-VLDL, y niveles más bajos de colesterol-HDL (Tabla 4). 
Tabla 4. Comportamiento del perfil lipídico en mujeres

\begin{tabular}{|c|c|c|c|c|c|c|}
\hline \multicolumn{2}{|c|}{ MUJERES } & $\begin{array}{l}\text { C-Total } \\
\text { (mg/dl) }\end{array}$ & $\begin{array}{l}\text { C-HDL } \\
(\mathrm{mg} / \mathrm{dI})\end{array}$ & $\begin{array}{l}\text { C-LDL } \\
(\mathrm{mg} / \mathrm{dl})\end{array}$ & $\begin{array}{c}\mathrm{TG} \\
(\mathrm{mg} / \mathrm{dl})\end{array}$ & $\begin{array}{l}\text { C-VLDL } \\
(\mathrm{mg} / \mathrm{dl})\end{array}$ \\
\hline \multirow{2}{*}{$\begin{array}{l}\text { Col.T } \\
\text { (mg/dl) }\end{array}$} & Pearson & 1 & 0,144 & $0,906^{* *}$ & $0,558 * *$ & $0,509 * *$ \\
\hline & Sig. (bilateral) & & 0,319 & 0,0001 & 0,0001 & 0,0001 \\
\hline \multirow{2}{*}{$\begin{array}{l}\text { C-HDL } \\
(\mathrm{mg} / \mathrm{dl})\end{array}$} & Pearson & 0,144 & 1 & $-0,007$ & $-0,285^{*}$ & $-0,341 *$ \\
\hline & Sig. (bilateral) & 0,319 & & 0,961 & 0,045 & 0,015 \\
\hline \multirow{2}{*}{$\begin{array}{l}\text { C-LDL } \\
(\mathrm{mg} / \mathrm{dl})\end{array}$} & Pearson & $0,906 * *$ & $-0,007$ & 1 & 0,271 & 0,219 \\
\hline & Sig. (bilateral) & 0,0001 & 0,961 & & 0,057 & 0,126 \\
\hline \multirow{2}{*}{$\begin{array}{l}\text { TG } \\
(\mathrm{mg} / \mathrm{dl})\end{array}$} & Pearson & $0,558^{* * *}$ & $-0,285^{*}$ & 0,271 & 1 & $0,978 * *$ \\
\hline & Sig. (bilateral) & 0,0001 & 0,045 & 0,057 & & 0,0001 \\
\hline \multirow{3}{*}{$\begin{array}{l}\text { C-VLDL } \\
(\mathrm{mg} / \mathrm{dl})\end{array}$} & Pearson & $0,509 * *$ & $-0,341^{\text {* }}$ & 0,219 & $0,978^{* * *}$ & 1 \\
\hline & Sig. (bilateral) & 0,0001 & 0,015 & 0,126 & 0,001 & \\
\hline & $\mathrm{N}$ & 50 & 50 & 50 & 50 & 50 \\
\hline
\end{tabular}

\section{Discusión}

La alimentación inadecuada, el tabaquismo, el alcoholismo y el sedentarismo, contribuye al desarrollo de la dislipidemia siendo un riesgo para el desarrollo de la aterosclerosis aumentando los casos de enfermedades cardiovasculares. ${ }^{21}$

En este sentido, si bien la mayoría de los encuestados refirió no tener el hábito de fumar, el consumo de bebidas alcohólicas sí se presentó en la mayoría de los casos. En el presente trabajo no se midió el consumo promedio de alcohol, destacando solo aquel grupo con mayor consumo siendo estos hombres. El excesivo consumo de alcohol se correlaciona en forma lineal con el aumento de los valores de presión arterial, de esta manera contribuye a la morbimortalidad por predisponer a ACV, también contribuye al aumento de la grasa corporal debido a su alto valor calórico (entre 100 y $200 \mathrm{kcal}$ ) incrementando el riesgo cardiovascular.

La frecuencia de consumo de frutas y verduras en hombres y mujeres fue de dos frutas por día cuatro veces a la semana y tres porciones de

${ }^{21}$ Escobar MC. Zacarías I.. 2014.

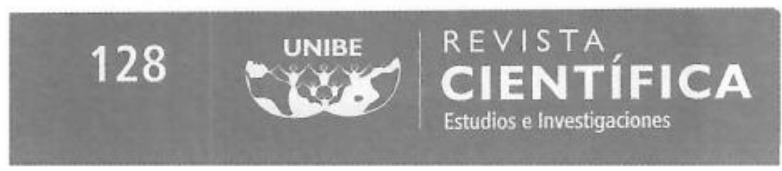


verduras por día cinco veces a la semana, cumpliéndolo recomendado por las guías alimentarias del Paraguay en cuanto a cantidad diaria, no así en cuanto a frecuencia de consumo de frutas y verduras, siendo adecuado un consumo diario de verduras y de al menos dos frutas por día. ${ }^{\square .22} \mathrm{Se}$ recomienda consumir distintas variedades de frutas y verduras ya que aportan vitaminas, minerales y fibras, principalmente de tipo soluble, las cuales mejoran el metabolismo de los carbohidratos, disminuyen el colesterol total y el colesterol LDL, importante para la prevención de enfermedades cardiovasculares.

Generalmente se acepta que el sedentarismo produce un incremento de enfermedades cardiovasculares, especialmente infarto de miocardio y muerte súbita en la población mundial siendo una de las principales causas prevenibles de mortalidad. Ello ha llevado a la mayoría de los expertos a incorporar a la actividad física como herramienta de manejo clínico y preventivo para la enfermedad cardiovascular. ${ }^{23},{ }^{24}$ Con respecto a lo mencionado y clasificándose como sedentaria a aquella persona que permanece sentado más de dos horas por día, se halló que a pesar de realizar algún tipo de actividad física, gran mayoría de los hombres como las mujeres presentan cierto índice de sedentarismo.

Analizando los factores de riesgo cardiovascular medidos, se encontraron valores significativamente superiores en hombres en lo que refiere a triglicéridos, colesterol-VLDL, presión arterial sistólica y diastólica y circunferencia de cintura. Si bien los hombres excedían mucho más los límites de circunferencia de cintura, misma tendencia se observó en las mujeres pero en menor medida presentando de igual manera riesgo cardiovascular, no así el colesterol-HDL que fue significativamente mayor en mujeres; sin embargo no se encontraron diferencias estadísticas en ambos sexos en cuanto al índice de masa corporal, colesterol total e índice de sedentarismo, lo cual es similar al trabajo publicado por Arsenault y cols ${ }^{25}$ realizado en Norfolk, Reino Unido donde se observó que los sujetos con circunferencia de cintura aumentada y triglicéridos

${ }^{22}$ Arteaga A, Bustos P, Soto R, Velasco N, Amigo H. Actividad física y su asociación con factores de riesgo cardiovascular. Un estudio en adultos jóvenes. RevMed Chile. 2010; 138: 1209-1216.

${ }^{23}$ Krämer V, et al., 2009.

${ }^{24}$ García E, Ortega F, Ruíz J, Meza J, Delgado M, González M, et al. El perfil lipídicometabólico en los adolescentes están más influidos por la condición física que por la actividad física (estudio AVENA). Rev. Esp. Cardiol. 2007; 60 (6): 581-8.

25 OMS Instrumento Versión Panamericana de STEPSwise (versión 2.0). Ginebra: Organización Mundial de la Salud; 2008. 
elevados tenían mayor índice de masa corporal y presentaban valores aumentados de la presión arterial en relación con los sujetos que tenían valores normales de circunferencia de cintura y de triglicéridos, también tenían alterado el perfil lipídico en general.

Existe un riesgo muy aumentado de enfermedad coronaria cuando la circunferencia de cintura juntamente con las concentraciones de triglicéridos se encuentra elevada, no así cuando solo uno de estos parámetros se encuentra aumentado siendo el riesgo menor.

Cabe mencionar que la hipertensión arterial aislada o asociada a otros factores de riesgo supone un factor de riesgo fundamental de padecer $\mathrm{ECV}^{26}$

En cuanto al comportamiento del perfil lipídico ante la intensidad, en general se encontró que a mayor intensidad de actividad física fueron significativamente mayores las concentraciones de colesterol-HDL, y significativamente menores las de los triglicéridos y colesterol-VLDL. Tanto en hombres como en mujeres se observó que a mayor intensidad de actividad física fueron significativamente mayores las concentraciones de colesterolHDL, no así las concentraciones de col-total, col-LDL, triglicéridos y colVLDL que fueron significativamente menores en los hombres.

Estos resultados concuerdan con el estudio mencionado por Texas Heart Institute, donde mencionan que antes de que inicie la menopausia, las mujeres tienen niveles más elevados de colesterol que los varones a expensas del colesterol-HDL debido a que el estrógeno aumenta los niveles de este en la sangre. La publicación de American Journal of Cardiology determina que los niveles de colesterol-HDL constituyen el factor más importante que predispone a una buena salud cardiovascular, por ello cuanto más elevado es el nivel de colesterol-HDL de la mujer, menor será la probabilidad de que sufra un episodio cardiovascular tal como un ataque cardíaco o cerebral. Pero después de la menopausia, los niveles de colesterol-HDL suelen bajar, incrementándose así el riesgo cardiovascular. Los niveles de colesterol-HDL y LDL pueden mejorarse con la dieta, la actividad física e hipolipemiantes si así se requiere. ${ }^{27}$

Debido a que los hombres carecen de un factor protector como lo es la hormona femenina estrógeno, necesitan realizar actividad física de mayor

\footnotetext{
${ }^{26}$ De la Maza MP, Díaz J, Gómez R, Maíz A, Darvich I, Ernst T, et al. Normas técnicas dislipidemias. División de Salud de las Personas. Departamento de Programas de las Personas. Programa Salud del Adulto. Chile. 2000. p. 1-81.

${ }^{27}$ Texas Heart Institute (Instituto del corazón de Texas). Factores de riesgo cardiovascular. Texas: Texas Heart Institute, Centro de información cardiovascular; 2011.
}

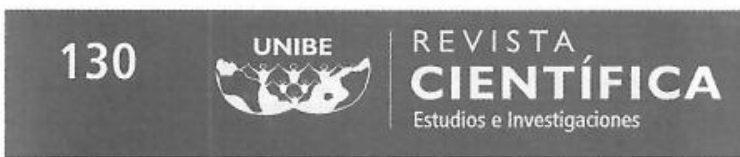


intensidad e incorporar mejores hábitos alimentarios como lo menciona un reciente estudio que concluyó que los cambios moderados de la dieta mejoran la concentración de colesterol y disminuye por tanto el riesgo de cardiopatía isquémica, solo cuando se sigue también un programa de ejercicios aeróbicos adecuados, entre los que se incluyen la caminata rápida, la natación, el ciclismo, entre otros, ya que son formas de realizar ejercicio, aumentar el colesterol-HDL, disminuir el colesterol-LDL, los triglicéridos, mejorando así el perfil lipídico. ${ }^{28}$

$\mathrm{Al}$ asociar la intensidad de la actividad física con el perfil lipídico según sexo, se observó que la mayoría de los hombres al igual que las mujeres $(\mathrm{H}=40 ; \mathrm{M}=46)$ realizaron actividad física de intensidad leve presentando los hombres en su mayoría niveles inadecuados de colesterol total, triglicéridos y colesterol-LDL, a diferencia de las mujeres que a la misma intensidad presentaron solo niveles de colesterol-LDL inadecuados.

Aintensidad moderada $(\mathrm{H}=7 ; \mathrm{M}=4)$, la mayoría de los hombres presentaron un perfil de colesterol-LDL inadecuado, no así las mujeres que a la misma intensidad presentaron en su mayoría el perfil lipídico adecuado.

Se observó que un hombre realizó actividad física intensa presentando un adecuado perfil lipídico. Boraita, A, refiere que la respuesta en el perfil lipídico después de un programa de actividad física así como los efectos es diferente según el tipo, la intensidad, frecuencia, duración y el tiempo de permanencia en el programa de entrenamiento físico. Si la actividad física se realiza a intensidades superiores al umbral anaeróbico conlleva al incremento de la concentración de lactato sanguíneo, esto facilita la recombinación de ácidos grasos libres para formar triglicéridos disminuyendo la disponibilidad de ácidos grasos libres como sustrato energético, siendo los hidratos de carbono la fuente principal de energía en la actividad física de elevadas intensidades; por otro lado los ácidos grasos son oxidados preferentemente en las fibras musculares oxidativas que son activadas durante la actividad física de intensidad moderada, entonces sobre esta base podría ser comprensible que no todo ejercicio $o$ actividad deportiva tenga los mismos efectos en el perfil lipídico. ${ }^{29,30}$

${ }^{8}$ IEDAR. Actividad física. Factor clave en la prevención de la obesidad. Informes sobre azúcar y alimentos azucarados $\mathrm{N}^{\circ} 3$. Instituto de estudios del azúcar y la remolacha.

${ }^{29}$ Maicas C, Lázaro E, Alcalá J, Hernández P, Rodríguez L. Etiología y fisiopatología de la hipertensión arterial esencial. Sociedad Castellana de Cardiología. 2003; 5 (3): 141-60.

${ }^{30}$ Hernández P, Mazo C, Ossa A. La autoprescripción del ejercicio físico en pacientes con diagnóstico de hipertensión arterial leve. Medellín: Instituto Universitario de Educación Física, Universidad de Antioquía; 2. 
La evidencia científica menciona de los efectos del ejercicio aeróbico de moderada intensidad en el perfil lipídico, entre los que hay que destacar el cambio favorable que ejerce en el control del metabolismo lipoproteico debido al incremento del colesterol-HDL por su carácter cardioprotector. Las modificaciones beneficiosas de los lípidos sanguíneos incluyen descensos del colesterol total, colesterol-LDL y triglicéridos ${ }^{31.32}$.

La evidencia científica que relaciona la actividad física y la salud cardiovascular muestra una disminución del riesgo con un gasto energético que varía entre 700 a 2000 Kcal por semana. Para lograr el objetivo de gastar al menos $700 \mathrm{Kcal}$ a la semana se debe realizar 30 minutos de caminata rápida o su equivalente por cinco días a la semana ${ }^{33}$.

Las mujeres con mayor colesterol total, presentaron una relación positiva y significativa con los valores de colesterol-LDL, triglicéridos y colesterol-VLDL. Aquellas mujeres con valores elevados de colesterolHDL presentaron una relación negativa y significativa con los valores de triglicéridos y colesterol-VLDL. Las que tuvieron valores elevados de colesterol-LDL, presentaron una relación positiva y significativa con los valores de colesterol total, las que presentaron valores elevados de triglicéridos presentaron una relación positiva y significativa con los valores de colesterol-total y de colesterol-VLDL, además de presentar una relación negativa y significativa con los valores de colesterol-HDL. Las mujeres con mayor colesterol-VLDL presentaron una relación positiva y significativa con los valores de colesterol-total y de triglicéridos, además de presentar una relación negativa y significativa con los valores de colesterol-HDL.

Los hombres con mayor colesterol total, presentaron una relación positiva y significativa con los valores de colesterol-LDL. Aquellos con valores elevados de colesterol-HDL presentaron una relación negativa y significativa con los valores triglicéridos y colesterol-VLDL. Los que tuvieron valores elevados de colesterol-LDL, presentaron una relación positiva y significativa con los valores de colesterol total. Los hombres con triglicéridos elevados presentaron una relación negativa y significativa con los valores de colesterol-HDL y una relación positiva y significativa con los valores de colesterol-VLDL, aquellos con mayor colesterol-VLDL presentaron una relación negativa y significativa con

\footnotetext{
${ }^{31}$ Djoussé L, Mukamal KJ. Consumo de alcohol y riesgo de hipertensión: ¿Tiene importancia el tipo de bebida o el patrón de consumo? Rev. Esp. Cardiol. 2009: 62(6): 603-5.

${ }^{32}$ Maicas C.. et al., 2003.

${ }^{33}$ Escobar MC, Zacarías I.. 2004
}

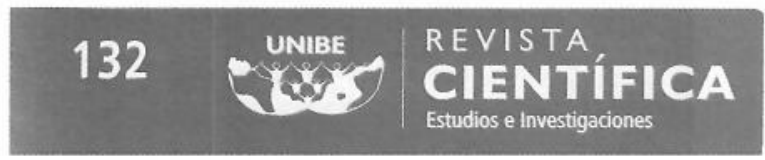


los valores de colesterol-HDL además de presentar una relación positiva y significativa con los valores de triglicéridos.

Gran parte de los sujetos presentaban dislipidemia, el cual se basa en niveles séricos aumentados de una o más fracciones lipídicas como el colesterol total, colesterol-LDL o triglicéridos y una disminución del nivel de colesterol-HDL, a su vez, los niveles de colesterol en sangre y su metabolismo están determinados en parte por las características genéticas del individuo y en parte por factores adquiridos tales como la dieta, el balance calórico y el nivel de actividad física. ${ }^{34}$

La hipertrigliceridemia grave es un factor de riesgo de aterosclerosis asociada a una mayor morbimortalidad coronaria lo que podría explicarse por su asociación muy frecuente con la disminución del colesterolHDL y por una modificación cualitativa del colesterol-LDL, estas se transforman en partículas más pequeñas y más densas que son las más susceptibles a la oxidación y por consiguiente más aterogénicas, así lo refiere el Programa de Salud del adulto.35

Los resultados del estudio si bien representan el tamaño muestral mínimo para lograr los fines de la investigación en la muestra estudiada; permiten concluir que se rechaza la hipótesis para la relación entre el gasto calórico de la actividad física y los niveles de colesterol HDL, triglicéridos y VLDL; respectivamente; no así con los niveles de colesterol-LDL y colesterol total; respectivamente.

Por lo cual, lo mencionado en diversas bibliografías sobre la actividad física si es realizada de manera constante además de tener efectos benéficos sobre el colesterol total, colesterol-HDL y colesterol-LDL también produce un incremento de la actividad lipolítica lo cual trae como consecuencia una disminución de los niveles plasmáticos de triglicéridos y de esta manera se puede prevenir factores de riesgos cardiovasculares asociados al sedentarismo como lo son las dislipidemias. ${ }^{36}$

Esto es ratificado mediante el estudio realizado por Leal y Cols, quienes explican que el mecanismo fisiológico exacto a través de la cual la actividad

\footnotetext{
34 Fundación del Corazón. Com. Fundación Española de Cardiología. Factores de riesgo cardiovascular. Málaga. Fundación Española del Corazón; [actualizada 27 ene 2011; citado 20 ago 2011]. Disponible en: http://www.fundaciondelcorazon.com/prevencion/riesgocardiovascular.html

3. Arsenault BJ, et al., 2010.

so Pontes LM, Pinheiro SS, Zemolin CM, Araujo TKC, Silva RL, Kumamoto FID, et al. Patrón de actividad física e influencia del sedentarismo en la ocurrencia de dislipidemias en adultos. Fit Perf J. 2008; 7(4):245-50.
} 
física disminuye la morbimortalidad de la enfermedad cardiovascular podría deberse a modificaciones en el perfil lipídico realizando ejercicios dinámicos, específicamente la disminución del colesterol-LDL y un incremento del colesterol-HDL. Esto podría ser favorable en relación a las personas que padecen diabetes mellitus ya que estos presentan como característica principal un perfil lipídico alterado con respecto a un aumento en los triglicéridos y del colesterol-VLDL y una disminución del colesterol-HDL. Las evidencias demuestran que el entrenamiento físico en estos pacientes, mejoran marcadamente la sensibilidad de la insulina en todo el organismo y la expresión de enzimas musculares reguladas por la misma. Así mismo la acumulación intramiocelular de lípidos, que está asociada a la insulinorresistencia en el músculo, puede disminuir agudamente, ya que se incrementa la oxidación de los ácidos grasos libres y los triglicéridos. Sin embargo, el mantenimiento de estos efectos parece necesitar la dedicación a un régimen de ejercicio regular y constante..$^{37,38}$

Hoy en día el sedentarismo es una epidemia en todo el mundo. La Organización Mundial de la Salud confirma que realizar actividad física de forma regular es uno de los principales componentes en la prevención del creciente aumento de enfermedades crónicas. Sin embargo, el $60 \%$ de la población en el mundo no llega a la recomendación mínima de realizar actividad física moderada durante 30 minutos al día. ${ }^{39}$

Se podría concluir entonces que la actividad física tiene un rol importante en la prevención, control y tratamiento de las enfermedades cardiovasculares por su influencia sobre varios factores de riesgo coronario, lo que constituye una medida costo/beneficio muy positiva, y es por todo ello que se insta a las personas a incorporar a su estilo de vida diario, al igual que una adecuada alimentación para prevenir desórdenes metabólicos y cardiovasculares a futuro.

${ }^{37}$ Arsenault BJ, et al., 2010.

${ }^{38}$ IEDAR. Actividad física. Factor clave en la prevención de la obesidad. Informes sobre azúcar y alimentos azucarados $\mathrm{N}^{\circ} 3$. Instituto de estudios del azúcar y la remolacha.

${ }^{39}$ Disponible en: http://texasheart.org/HIC/Topics_Esp/HSmart/riskspan.cfm

\section{UNIBE $\begin{aligned} & \text { REVISTA } \\ & \text { CIENTÍFICA }\end{aligned}$}




\section{BIBLIOGRAFÍA}

Arsenault BJ, Lemieux I, Després JP. Fentotipo hipertrigliceridemiacintura aumentada y riesgo coronario. 2010. Disponible en: http://www. intramed.net/contenidover.asp? contenidoID $=66730$

Arteaga A, Bustos P, Soto R, Velasco N, Amigo H. Actividad física y su asociación con factores de riesgo cardiovascular. Un estudio en adultos jóvenes. RevMed Chile. 2010; 138: 1209-1216.

Barreda R, Santos J, Sakuray S, Villanueva J, Delgado I, Avendaño M. Prevalencia de factores de riesgo de enfermedades cardiovasculares en mayores de 18 años de los distritos de Tacna, Gregorio Alabarracin, Ciudad Nueva, Tarata y Candarave 2007; (78): 1-43.

Boraita A. La práctica deportiva mejora el perfil lipídico plasmático, pero ¿a cualquier intensidad? Rev. Esp. Cardiol. 2004; 57 (6): 495-498.

De la Maza MP, Díaz J, Gómez R, Maíz A, Darvich I, Ernst T, et al. Normas técnicas dislipidemias. División de Salud de las Personas. Departamento de Programas de las Personas. Programa Salud del Adulto. Chile. 2000. p. 1-81.

Djoussé L, Mukamal KJ. Consumo de alcohol y riesgo de hipertensión: ¿Tiene importancia el tipo de bebida o el patrón de consumo? Rev. Esp. Cardiol. 2009; 62(6): 603-5.

Escobar MC, Zacarías I. Programa de actividad física para la prevención y control de los factores de riesgo cardiovascular. Instituto de Nutrición y Tecnología de los Alimentos (INTA) de la Universidad de Chile, Comité de Prevención de Riesgo Cardiovascular de la Sociedad de Cardiología y Cirugía Cardiovascular y la Sociedad de Kinesiología en Cardiología y Cirugía Cardiovascular, Organización Panamericana de la Salud/ Organización Mundial de la Salud. Santiago. 2004.

Fundación del corazón. Com. Fundación Española de Cardiología. Factores de riesgo cardiovascular. Málaga. Fundación Española del Corazón; [actualizada 27 ene 2011; citado 20 ago 2011]. Disponible en: http://www.fundaciondelcorazon.com/prevencion/riesgocardiovascular.html

García E, Ortega F, Ruíz J, Meza J, Delgado M, González M, et al. El perfil lipídico-metabólico en los adolescentes están más influidos por la condición física que por la actividad física (estudio AVENA). Rev. Esp. Cardiol. 2007; 60 (6): 581-8. 
Hernández MA, García HL. Factores de riesgo y protectores de enfermedades cardiovasculares en población estudiantil universitaria. Rev. Fac. Med. 2007; 30 (2): 119-123.

Hernández P, Mazo C, Ossa A. La autoprescripción del ejercicio físico en pacientes con diagnóstico de hipertensión arterial leve. Medellín: Instituto Universitario de Educación Física, Universidad de Antioquía; 2005.

IEDAR. Actividad física. Factor clave en la prevención de la obesidad. Informes sobre azúcar y alimentos azucarados $\mathrm{N}^{\circ} 3$. Instituto de estudios del azúcar y la remolacha.

Imizcoz MA.La hipertensión arterial como factor de riesgo de enfermedad cardiovascular. Anales de la facultad de Navarra; 21 (1).

INAN-MSPBS. Guías Alimentarias del Paraguay. Asunción-Paraguay: Ministerio de Salud Pública y Bienestar Social. 2003.

Krämer V,Acevedo M, Orellana L, Chamorro G. Corbalán R. Bustamante MJ, et al. Actividad Física y potencia aeróbica: ¿Cómo influyen sobre los factores de riesgo cardiovascular clásicos y emergentes? Rev. Méd. Chile. 2009; 137: 737-745.

Leal E, Aparicio D, Luti Y, Acosta L. Finol F. Rojas E. et al. Actividad física y enfermedad cardiovascular. Revista Latinoamericana de Hipertensión. 2009; 4 (1): 3-17.

Maicas C, Lázaro E, Alcalá J, Hernández P. Rodríguez L. Etiología y fisiopatología de la hipertensión arterial esencial. Sociedad Castellana de Cardiología. 2003; 5 (3): 141-60.

Ministerio de Salud Pública y Bienestar Social. Enfermedades cardiovasculares constituirán 30\% de causas de muerte en el 2020, según estimaciones del INPCARD. Disponible en: http://www.mspbs.gov.py

OMS Instrumento Versión Panamericana de STEPSwise (versión 2.0). Ginebra: Organización Mundial de la Salud: 2008.

OMS. Enfermedades cardiovasculares. Ginebra: Organización Mundial de la Salud; 2011.

Onzari M. Fundamentos de Nutrición en el Deporte. $1^{\mathrm{a}}$ ed. Buenos Aires: El Ateneo; 2008. p. 328.

Organización Panamericana de la Salud. Consulta regional: prioridades para la salud cardiovascular en las Américas. Mensajes claves para los

\section{UNIBE ${ }^{\text {REVISTA }}$ CIENTÍFICA}


decisores. Ordúñez-García P, Campillo-Artero C, eds. Washington, D.C.: OPS; 2011.

Pontes LM, Pinheiro SS, Zemolin CM, Araujo TKC, Silva RL, Kumamoto FID, et al. Patrón de actividad física e influencia del sedentarismo en la ocurrencia de dislipidemias en adultos. Fit Perf J. 2008; 7(4):245-50.

Texas Heart Institute (Instituto del corazón de Texas). Factores de riesgo cardiovascular. Texas: Texas Heart Institute, Centro de información cardiovascular; 2011.

Ulate G, Fernández A. Relaciones del perfil lipídico con variables dietéticas, antropométricas, bioquímicas y otros factores de riesgo cardiovascular en estudiantes universitarios. AMC. 2001; 43(2): 70-6. 
138 UNIBE REVISTA CIENTÍFICA Estudios e Investigaciones 